\title{
Damping rate of a fermion in a medium
}

\author{
J. C. D'Olivo* \\ Instituto de Ciencias Nucleares \\ Universidad Nacional Autónoma de México \\ Apartado Postal 70-543, 04510 México, D.F., México \\ José F. Nieves ${ }^{\dagger}$ \\ Laboratory of Theoretical Physics \\ Department of Physics, P. O. Box 23343 \\ University of Puerto Rico \\ Río Piedras, Puerto Rico 00931-3343
}

(September 1994)

\begin{abstract}
We examine the relation between the damping rate of a massless, chiral fermion that propagates in a medium, and the rate $\Gamma$ of approach to equilibrium. It is proven that these quantities are equal, by showing that they are given by the same formula in terms of the imaginary part of the self-energy evaluated at the energy of the propagating fermion mode. This result is valid provided $\Gamma$ is defined by using the appropriate wave functions of the mode.
\end{abstract}

*Partially supported by Grant No. DGAPA-IN100691

†Partially supported by the US National Science Foundation Grant PHY-9320692 
It is well known that a massless fermion that propagates through a medium acquires a dispersion relation that differs from that in the vacuum [1]. In general, the dispersion relation of a fermion with momentum $p^{\mu}=(\varepsilon, \vec{P})$ is not given by $\varepsilon_{P}=P$ and, in particular, $\varepsilon_{P}$ is not zero at zero momentum. Writing

$$
\varepsilon_{P}=\varepsilon_{r}-i \frac{\gamma}{2}
$$

with $\varepsilon_{r}$ and $\gamma$ being real, the quantity $M=\varepsilon_{r}(P=0)$ can be interpreted as an effective mass, and $\gamma$ as the fermion damping rate [2]. In the framework of Finite Temperature Field Theory (FTFT) [3, [4, the quantity of fundamental interest is the self-energy, from which the dispersion relation is determined.

Besides the fermion damping rate, a distinct concept, also related to the imaginary part of the self-energy, has been used in the literature. This quantity, to which we will refer as the total reaction rate, is denoted by $\Gamma$ and its inverse gives the time scale for a distribution function to approach its equilibrium value [5],6]. In Ref. [5], Weldon expressed $\Gamma$ as a combination of probabilities amplitudes for various processess, weighted by certain statistical factors that account for the absortion and emission of the particles by the medium. By analysing the one-loop contributions to boson and fermion self-energies, he showed that $\Gamma$ could be calculated from the imaginary part of the self-energy [7], although no attempt was made to connect it with the damping rate.

In this manner one ends up with two apparently different physical quantities, the damping $\gamma$ and the total reaction rate $\Gamma$, both related to the imaginary part of the self-energy. By studying the linear response of a medium to an external field, the equality between $\Gamma$ and $\gamma$ was established for the collective color modes in a QCD plasma [8]. However, to our knowledge the same relation has not been derived for the fermion case, and this fact has been the source of some confussion. In a recent article on the subject [9], two different formulas are used by the authors to compute the damping and the total reaction rate in terms of the self-energy (Eqs. (2.2) and (2.27) of Ref. [9]). Our aim in this work is to show that no such dichotomy is necessary and, when correctly calculated in terms of the fermion 
self-energy, the damping and the total rate are given by the same formula. In this fashion, the result $\gamma=\Gamma$ is proved to hold also for fermions.

Calculations of $\gamma$ carried out during the last few years within the realm of hot gauge theories, have produced contradictory results, entangled by questions of gauge invariance and other problems [10]. It has been pointed out that such ambiguities are a sympton of an incomplete calculation, and a resummation of higher order corrections is necessary to get the correct result [11]. To avoid these complications and make our presentation as clear as possible, we examine here the simpler but still instructive situation of fermions interacting with a scalar field through a Yukawa interaction.

We begin by obtaining a formula for $\gamma$ in terms of the self-energy, which is valid when $\gamma \ll \varepsilon_{r}$. This is the physically interesting regime since otherwise the system would be overdamped and the concept of a propagating mode is not meaningful. That formula, derived by expanding the equation for the dispersion relation up to terms that are at most linear in $\gamma$ and the absortive part of the self-energy, is the proper expression for the fermion damping rate and it corrects or complements formulas that have been quoted in previous works [9, 12]. We then proceed to demonstrate that the correct formula for $\gamma$ coincides with $\Gamma$, provided the latter quantity is defined in terms of the transition probabilities calculated with the properly normalized spinors that satisfy the effective Dirac equation in the medium instead of the free-particle spinors. The equality between the damping and the total rate is first illustrated with a one-loop calculation, and then a general proof is given using the spectral representation of the fermion self-energy.

The properties of a particle that propagates through a medium are determined by the linear part of the effective (classical) field equation. For a massless fermion with momentum $p^{\mu}$, the equation, in momentum space, is

$$
\left(\not p-\Sigma_{e f f}\right) \psi=0
$$

with the self-energy $\Sigma_{e f f}$ having the form

$$
\Sigma_{e f f}=a \not p+b \mu,
$$


where $a$ and $b$ are functions of the variables

$$
\begin{aligned}
\varepsilon & =p \cdot u, \\
P & =\sqrt{\varepsilon^{2}-p^{2}},
\end{aligned}
$$

which give the energy and the magnitude of the 3-momentum $\vec{P}$ in the rest frame of the medium. We have introduced the vector $u^{\mu}$ representing the velocity 4 -vector of the medium which in its own rest frame has components $(1, \overrightarrow{0})$.

The self-energy can be decomposed as

$$
\Sigma_{e f f}=\Sigma_{r}+i \Sigma_{i},
$$

in terms of the absorptive and dispersive parts

$$
\begin{aligned}
\Sigma_{r} & =\frac{1}{2}\left(\Sigma_{e f f}+\bar{\Sigma}_{e f f}\right), \\
\Sigma_{i} & =\frac{1}{2 i}\left(\Sigma_{e f f}-\bar{\Sigma}_{e f f}\right),
\end{aligned}
$$

with $\bar{\Sigma}_{e f f}=\gamma^{0} \Sigma_{e f f}^{\dagger} \gamma^{0}$. Within the framework of the real-time formulation of FTFT, the dispersive part of the self-energy is given by

$$
\Sigma_{r}=\Sigma_{11 r}
$$

while the absorptive part can be calculated by any of the following formulas

$$
\begin{aligned}
\Sigma_{i} & =\frac{\Sigma_{11 i}}{1-2 n_{F}(x)} \\
& =\frac{i}{2}\left(\Sigma_{21}-\Sigma_{12}\right) \\
& =\frac{\Sigma_{12}}{2 i n_{F}(x)} .
\end{aligned}
$$

The $\Sigma_{a b}$ are the elements of the $2 \times 2$ self-energy matrix to be computed using the Feynman rules of the theory. In Eq. (8),

$$
n_{F}(x)=\frac{1}{e^{x}+1}
$$


is the Fermi-Dirac distribution for the fermion that propagates in the medium, written in terms of the variable

$$
x=\beta(p \cdot u-\mu),
$$

where $\beta$ is the inverse temperature and $\mu$ is the chemical potential of the fermion.

The dispersion relations of the particle and antiparticle are determined by requiring Eq. (2) to have non-trivial solutions. The corresponding condition determines also the poles of the fermion propagator and can be written in the form

$$
f(\varepsilon, P) \bar{f}(\varepsilon, P)=0
$$

where

$$
\begin{aligned}
& f(\varepsilon, P) \equiv(1-a)(\varepsilon-P)-b, \\
& \bar{f}(\varepsilon, P) \equiv(1-a)(\varepsilon+P)-b,
\end{aligned}
$$

with $a$ and $b$ defined in Eq. (3). Then, the dispersion relations $\varepsilon_{P}$ are obtained as the solutions of

$$
f\left(\varepsilon_{P}, P\right)=0
$$

and

$$
\bar{f}\left(\varepsilon_{P}, P\right)=0 .
$$

In general, the solutions $\varepsilon_{P}$ are complex, and a consistent interpretation in terms of the dispersion relation for a mode propagating through a medium is possible only if the imaginary part of $\varepsilon_{P}$ is small compared to its real part. In this case the mode can be visualized as a particle with an energy and a damping rate given by the real and imaginary part of $\varepsilon_{P}$, respectively [13].

Under such assumptions, each one of Eqs. (13) and (14) yields two distinct solutions, one with positive energy and the other with negative energy, whose physical interpretation 
has been discussed in detail in Ref. ( [14]). Here we will concentrate on the solution of Eq. (13) having a positive real part, which corresponds to the particle mode with energy $\varepsilon_{r}$, but similar considerations and results apply to the other solutions.

It is convenient to separate the function $f$ into its dispersive and absorptive parts, and write [15]

$$
f(\varepsilon, P)=f_{r}(\varepsilon, P)+i f_{i}(\varepsilon, P)
$$

with a similar decomposition for $a$ and $b$. Then

$$
\begin{aligned}
& f_{r}=\left(1-a_{r}\right)(\varepsilon-P)-b_{r}, \\
& f_{i}=-a_{i}(\varepsilon-P)-b_{i} .
\end{aligned}
$$

Writing $\varepsilon_{P}$ in the form of Eq. (1), the condition (13) becomes

$$
f_{r}\left(\varepsilon_{r}-i \frac{\gamma}{2}, P\right)+i f_{i}\left(\varepsilon_{r}-i \frac{\gamma}{2}, P\right)=0
$$

which we solve by expanding in powers of $\gamma$ and retaining only terms that are at most linear in $\gamma$ and $f_{i}$. Thus, $\varepsilon_{r}$ is determined as the solution of

$$
f_{r}\left(\varepsilon_{r}, P\right)=0
$$

while $\gamma$ is given by the formula

$$
\frac{\gamma}{2}=\frac{f_{i}\left(\varepsilon_{r}, P\right)}{N_{P}}
$$

where

$$
N_{P}=\left[\frac{\partial f_{r}}{\partial \varepsilon}\right]_{\varepsilon=\varepsilon_{r}} .
$$

It is important to observe that the quantity $N_{P}$ coincides with the normalization factor that has to be taken into account in order to construct the spinors representing the one-particle states, and which need to be included to correctly calculate the probability amplitudes for the various processes involving the fermion [16]. We also want to remark that our Eqs. 
(18) and (19), with the factor $N_{P}$ included, are analogous to those used in the context of many-body physics 17.

The one-particle states have associated the wave functions $u_{L, R}$ satisfying the Dirac equation

$$
\left(\not p-\Sigma_{r}\right) u_{L, R}=0
$$

obtained by discarding the absorptive part of the self-energy. While the explicit solutions of this equation are more easily worked out in the rest frame of the medium, for our present purposes the knowledge of the spinor projection operator will be sufficient. For the negativehelicity solution it is given by

$$
u_{L} \bar{u}_{L}=\frac{\varepsilon_{r}}{N_{P}} L \not h
$$

where, as usual, $L=\left(1-\gamma_{5}\right) / 2, N_{P}$ has been defined in Eq. (20) and

$$
n^{\mu}=\frac{1}{P}\left(p^{\mu}-\left(\varepsilon_{r}-P\right) u^{\mu}\right)
$$

is a null vector, whose components in the rest frame of the medium are $n^{\mu}=\left(1, \frac{\vec{P}}{P}\right)$. There is a relation for the positive-helicity solution $u_{R}$ similar to Eq. (22). For definiteness, in the rest of the article we will work with the negative-helicity solution, but exactly the same considerations and results apply to the other one as well. Using

$$
\Sigma_{i}=a_{i} \not p+b_{i} \psi
$$

and Eq. (22), it is easy to verify by direct computation that the formula for the damping rate given in Eq. (19) can be written in the form

$$
\begin{aligned}
\gamma & =-\frac{1}{2 N_{P}} \operatorname{Tr}\left[\not h \Sigma_{i}\left(\varepsilon_{r}, P\right)\right] \\
& =-\frac{1}{\varepsilon_{r}} \bar{u}_{L} \Sigma_{i}\left(\varepsilon_{r}, P\right) u_{L} .
\end{aligned}
$$

In Eq. (25) we have indicated explicitly that $\Sigma_{i}$ must be evaluated at $\varepsilon=\varepsilon_{r}$. This a convenient formula for $\gamma$ that will allow us to identify it with the rate $\Gamma$ computed by squaring 
amplitudes and integrating over the phase space with the appropriate statistical weights. One should become aware of the general nature of Eq. (25), which we have derived under the assumption that the damping is small, but otherwise not tied in anyway to a perturbative, or for that matter any other, particular method of calculation of the self-energy.

If $\varepsilon_{r}$ and $N_{P}$ are approximated by their value in vacuum $\left(\varepsilon_{r} \simeq P\right.$ and $\left.N_{P} \simeq 1\right)$, then it is easy to see from Eq. (19) that $\gamma \simeq-2 \operatorname{Im} b(P, P)$, which coincides with the formula given in Ref. ( 12]) for the damping rate in the high momentum limit $(P \gg T)$. However, in other momentum regimes

$$
\gamma \neq-2 \operatorname{Im} b\left(\varepsilon_{r}, P\right)
$$

and the damping rate must be calculated as given in Eq. (19) (or Eq. (25)), and by using the true dispersion relation in the medium $\varepsilon_{r}$. In particular, the formula for the damping rate quoted in Eq. (2.27) of Ref. ( [9]), which is equivalent to put $\gamma=-2 \operatorname{Im} b\left(\varepsilon_{r}, P\right)$, does not correspond to the imaginary part of the energy momentum-relation and does not coincide with the total reaction rate.

The next step is to establish the relation between $\Sigma_{i}$ and the total reaction rate $\Gamma$, which we will do first by using a 1-loop example calculation of $\Sigma_{i}$. As an instructive example, that is free of the ambiguities associated with gauge invariance pointed out in the beginning, we adopt the model of a chiral fermion field $f_{L}$ interacting with a scalar field $\phi$ and a massive fermion $\psi$ according to

$$
L_{\mathrm{int}}=\lambda \bar{\psi}_{R} f_{L} \phi+h . c .
$$

The simplest way to proceed with the calculation is to compute $\Sigma_{12}$ and then use Eq. (8). The relevant diagram is shown in Fig. 1 and, according to the Feynman rules, its contribution is given by

$$
-i \Sigma_{12}(p)=(i \lambda)(-i \lambda) \int \frac{d^{4} p^{\prime}}{(2 \pi)^{4}} i \Delta_{21}\left(p^{\prime}-p\right) \operatorname{RiS}_{12}\left(p^{\prime}\right) L
$$

The components of the propagator matrices for the massive fermion and the scalar are 


$$
\begin{aligned}
& S_{12}\left(p^{\prime}\right)=2 \pi i \delta\left(p^{\prime 2}-m_{\psi}^{2}\right)\left[\eta_{F}\left(p^{\prime}\right)-\theta\left(-p^{\prime} \cdot u\right)\right]\left(\not p^{\prime}+m_{\psi}\right) \\
& \Delta_{21}(k)=-2 \pi i \delta\left(k^{2}-m_{\phi}^{2}\right)\left[\eta_{B}(k)+\theta(k \cdot u)\right]
\end{aligned}
$$

where, in terms of the variables

$$
\begin{gathered}
x^{\prime}=\beta\left(p^{\prime} \cdot u-\mu_{\psi}\right), \\
x_{\phi}=\beta\left(k \cdot u-\mu_{\phi}\right),
\end{gathered}
$$

we have

$$
\begin{aligned}
& \eta_{F}\left(p^{\prime}\right)=\theta\left(p^{\prime} \cdot u\right) n_{F}\left(x^{\prime}\right)+\theta\left(-p^{\prime} \cdot u\right) n_{F}\left(-x^{\prime}\right), \\
& \eta_{B}(k)=\theta(k \cdot u) n_{B}\left(x_{\phi}\right)+\theta(-k \cdot u) n_{B}\left(-x_{\phi}\right),
\end{aligned}
$$

with $\theta$ being the step function. The fermion distribution $n_{F}$ is given in Eq. (9), while for the scalar

$$
n_{B}\left(x_{\phi}\right)=\frac{1}{e^{x_{\phi}}-1}
$$

Momentum as well as charge and lepton number conservation imply that

$$
x^{\prime}-x=x_{\phi}
$$

where $x$ has been defined in Eq. (10).

The propagators $S_{12}$ and $\Delta_{21}$ can be rewritten using the identities

$$
\begin{aligned}
\eta_{F}\left(p^{\prime} \cdot u\right)-\theta\left(-p^{\prime} \cdot u\right) & =\epsilon\left(p^{\prime} \cdot u\right) n_{F}\left(x^{\prime}\right), \\
\eta_{B}(k \cdot u)+\theta(k \cdot u) & =\epsilon(k \cdot u) n_{B}\left(x_{\phi}\right) e^{x_{\phi}},
\end{aligned}
$$

where $\epsilon(x)=\theta(x)-\theta(-x)$. Substituting the resulting formulas into Eq. (27) and then using Eq. (8), we obtain

$$
\begin{aligned}
\Sigma_{i}= & -\frac{\lambda^{2}}{8 \pi^{2}} \int d^{4} p^{\prime} \delta\left(p^{2}-m_{\psi}^{2}\right) \delta\left[\left(p^{\prime}-p\right)^{2}-m_{\phi}^{2}\right] \\
& \times \epsilon\left(p^{\prime} \cdot u\right) \epsilon\left(\left(p^{\prime}-p\right) \cdot u\right)\left(n_{F}\left(x^{\prime}\right)+n_{B}\left(x_{\phi}\right)\right) R\left(\not p^{\prime}+m_{\psi}\right) L .
\end{aligned}
$$


To derive this result we used the equality

$$
e^{x_{\phi}} n_{F}\left(x^{\prime}\right) n_{B}\left(x_{\phi}\right)=n_{F}(x)\left(n_{F}\left(x^{\prime}\right)+n_{B}\left(x_{\phi}\right)\right),
$$

which is a consequence of Eq. (32). The integral in Eq. (34) can be evaluated for different cases, but here we do not go any further in that direction. For our purposes it is more convenient to express Eq. (34) as

$$
\begin{aligned}
\Sigma_{i}= & -\frac{\lambda^{2}}{8 \pi^{2}} \int \frac{d^{3} p^{\prime}}{2 E^{\prime}} \frac{d^{3} k}{2 \omega_{k}} R\left[\delta^{(4)}\left(p+k-p^{\prime}\right)\left(n_{\psi}+n_{\phi}\right)\left(\not p^{\prime}+m_{\psi}\right)\right. \\
& +\delta^{(4)}\left(p-k+p^{\prime}\right)\left(\bar{n}_{\psi}+\bar{n}_{\phi}\right)\left(\not \not^{\prime}-m_{\psi}\right) \\
& +\delta^{(4)}\left(p-k-p^{\prime}\right)\left(1-n_{\psi}+\bar{n}_{\phi}\right)\left(\not p^{\prime}+m_{\psi}\right) \\
& \left.+\delta^{(4)}\left(p+k+p^{\prime}\right)\left(1-\bar{n}_{\psi}+n_{\phi}\right)\left(\not p^{\prime}-m_{\psi}\right)\right] L,
\end{aligned}
$$

where $n_{\psi}$ and $n_{\phi}$ are the particle density distributions

$$
\begin{aligned}
& n_{\psi}=\frac{1}{e^{\beta\left(p^{\prime} \cdot u-\mu_{\psi}\right)}+1}, \\
& n_{\phi}=\frac{1}{e^{\beta\left(k \cdot u-\mu_{\phi}\right)}-1},
\end{aligned}
$$

and $\bar{n}_{\psi, \phi}$ are the respective antiparticle distributions, obtained from $n_{\psi, \phi}$ by changing the sign of the chemical potential. In addition,

$$
\begin{aligned}
p^{\prime \mu} & =\left(E^{\prime}, \vec{p}^{\prime}\right), \\
k^{\mu} & =\left(\omega_{k}, \vec{k}\right),
\end{aligned}
$$

with

$$
\begin{aligned}
& E^{\prime}=\sqrt{\vec{p}^{2}+m_{\psi}^{2}}, \\
& \omega_{k}=\sqrt{\vec{k}^{2}+m_{\phi}^{2}}
\end{aligned}
$$

Now, by means of the projection operators

$$
\begin{aligned}
& \sum_{s} u_{\psi}\left(p^{\prime}, s\right) \bar{u}_{\psi}\left(p^{\prime}, s\right)=\not p^{\prime}+m_{\psi}, \\
& \sum_{s} v_{\psi}\left(p^{\prime}, s\right) \bar{v}_{\psi}\left(p^{\prime}, s\right)=\not p^{\prime}-m_{\psi},
\end{aligned}
$$


Eq. (35) leads to the relation

$$
\bar{u}_{L} \Sigma_{i}\left(\varepsilon_{r}, P\right) u_{L}=-\varepsilon_{r} \Gamma
$$

where we have defined

$$
\begin{aligned}
\Gamma \equiv & \frac{1}{2 \varepsilon_{r}} \int \frac{d^{3} k}{(2 \pi)^{3} 2 \omega_{k}} \frac{d^{3} p^{\prime}}{(2 \pi)^{3} 2 E^{\prime}}(2 \pi)^{4} \\
& \times\left\{\delta^{(4)}\left(p+k-p^{\prime}\right)\left[n_{\psi}\left(1+n_{\phi}\right)+n_{\phi}\left(1-n_{\psi}\right)\right] \sum_{s}\left|M_{I}\right|^{2}\right. \\
& +\delta^{(4)}\left(p-k+p^{\prime}\right)\left[\bar{n}_{\psi}\left(1+\bar{n}_{\phi}\right)+\bar{n}_{\phi}\left(1-\bar{n}_{\psi}\right)\right] \sum_{s}\left|M_{I I}\right|^{2} \\
& +\delta^{(4)}\left(p-k-p^{\prime}\right)\left[\left(1-n_{\psi}\right)\left(1+\bar{n}_{\phi}\right)+n_{\psi} \bar{n}_{\phi}\right] \sum_{s}\left|M_{I}\right|^{2} \\
& \left.+\delta^{(4)}\left(p+k+p^{\prime}\right)\left[\left(1-\bar{n}_{\psi}\right)\left(1+n_{\phi}\right)+\bar{n}_{\psi} n_{\phi}\right] \sum_{s}\left|M_{I I}\right|^{2}\right\}
\end{aligned}
$$

and

$$
\begin{gathered}
M_{I}=\lambda \bar{u}_{\psi}\left(p^{\prime}, s\right) u_{L}(p), \\
M_{I I}=\lambda \bar{v}_{\psi}\left(p^{\prime}, s\right) u_{L}(p) .
\end{gathered}
$$

The formula for $\Gamma$ given in Eq. (39) is immediately recognized as the total rate for a particle of energy $\varepsilon_{r}$ and momentum $P$ (as seen from the rest frame of the medium) with integrations over the phase space weighted by the statistical factors appropriate for each process. $M_{I}$ is the amplitude for $f \phi \rightarrow \psi$ or the decay $f \rightarrow \psi \bar{\phi}$, while $M_{I I}$ is the amplitude for $f \bar{\psi} \rightarrow \phi$ or $f \phi \bar{\psi} \rightarrow 0$. The amplitudes for the inverse reactions are given by the complex conjugates of $M_{I}$ and $M_{I I}$. For specific values of $\varepsilon_{r}$ and $P$, some of these processes will be kinematically forbidden and will not contribute to $\Gamma$. As is well known [18], for Fermi systems the inverse reactions are inhibited as a consequence of the Pauli blocking effect, and they contribute additively to the depletion of the state. Therefore, in this case $\Gamma$ is given by the sum of the rates for the direct and inverse processes, instead of their difference as in the bosonic case. Although our expression for $\Gamma$ in Eq. (39) has the same form as the one used in Ref. [5], it is important to keep in mind that for us, the propagating fermion is represented in the amplitudes $M_{I, I I}$ by the spinor $u_{L}$ that obeys the effective Dirac equation 
in the medium, and not by a free particle spinor. Comparing Eqs. (25) and (38) we finally obtain the relation

$$
\gamma=\Gamma
$$

We next show that the relation between the matrix element of the absorptive part $\Sigma_{i}$ of the self-energy and the total rate, that was established above by looking at the 1-loop calculation, is a general result. For this purpose we recall that, in the real-time formulation of FTFT, the self-energy of a fermion in a thermal background is a $2 \times 2$ matrix whose elements are defined by

$$
\begin{aligned}
i \Sigma_{21}(z-y)_{\alpha \beta} & =-\left\langle\eta_{\alpha}(z) \bar{\eta}_{\beta}(y)\right\rangle \\
i \Sigma_{12}(z-y)_{\alpha \beta} & =\left\langle\bar{\eta}_{\beta}(y) \eta_{\alpha}(z)\right\rangle \\
-\Sigma_{11}(z-y) & =\Sigma_{21}(z-y) \theta\left(z^{0}-y^{0}\right)+\Sigma_{12}(z-y) \theta\left(y^{0}-z^{0}\right), \\
-\Sigma_{22}(z-y) & =\Sigma_{21}(z-y) \theta\left(y^{0}-z^{0}\right)+\Sigma_{12}(z-y) \theta\left(z^{0}-y^{0}\right),
\end{aligned}
$$

where $\eta$ and $\bar{\eta}$ are the fermion source fields, in terms of which the interaction Lagrangian is

$$
L_{\text {int }}=\bar{f}_{L} \eta+\bar{\eta} f_{L}
$$

For the scalar model of Eq. (26) $\eta=\lambda \phi^{*} \psi_{R}$. The angle brackets in Eq. (42) stand for the statistical average which, for any operator $\mathcal{O}$, is defined by

$$
\langle\mathcal{O}\rangle=\frac{1}{Z} \sum_{i}\langle i|\rho \mathcal{O}| i\rangle
$$

where

$$
\rho=e^{-\beta H+\sum_{A} \alpha_{A} Q_{A}}
$$

and

$$
Z=\sum_{i}\langle i|\rho| i\rangle,
$$


with $H$ being the Hamiltonian of the system. The quantities $Q_{A}$ are the (conserved) charges that commute with $H$, and the $\alpha_{A}$ are the chemical potentials that characterize the composition of the background.

Using the convention of Eq. (43), the amplitude for the decay $n+f(p) \rightarrow m$, where $n$ and $m$ label any two states of the system, is

$$
A=(2 \pi)^{4} \delta^{(4)}\left(p+q_{n}-q_{m}\right)\left\langle m\left|\bar{\eta}(0) u_{L}(p)\right| n\right\rangle,
$$

where $u_{L}(p)$ is the properly normalized spinor of the propagating mode $f$. From this follows that the total decay rate, averaged over the initial states of the system, is

$$
\Gamma_{D}=\frac{1}{Z} \frac{1}{2 p^{0}} \sum_{n, m}\left|\left\langle m\left|\bar{\eta}(0) u_{L}(p)\right| n\right\rangle\right|^{2}(2 \pi)^{4} \delta^{(4)}\left(p+q_{n}-q_{m}\right) Z_{n},
$$

where $Z$ is defined in Eq. (46) and

$$
\begin{aligned}
Z_{n} & =\langle n|\rho| n\rangle \\
& =e^{-\beta q_{n} \cdot u+\alpha_{n}} .
\end{aligned}
$$

Here $\alpha_{n}$ is the eigenvalue of the operator $\hat{\alpha}=\sum_{A} \alpha_{A} Q_{A}$ corresponding to the state $|n\rangle$; i.e., $\hat{\alpha}|n\rangle=\alpha_{n}|n\rangle$. In similar fashion, the total probability for the inverse decay $m \rightarrow n+f(p)$ is given by

$$
\int \frac{d^{3} p}{(2 \pi)^{3}} \Gamma_{I}
$$

where

$$
\Gamma_{I}=\frac{1}{Z} \frac{1}{2 p^{0}} \sum_{n, m}\left|\left\langle n\left|\bar{u}_{L}(p) \eta(0)\right| m\right\rangle\right|^{2}(2 \pi)^{4} \delta^{(4)}\left(p+q_{n}-q_{m}\right) Z_{m}
$$

Since $\eta$ and $f$ have the same quantum numbers, then

$$
\left\langle n\left|\bar{u}_{L}(p) \eta(0)\right| m\right\rangle \neq 0
$$

only for those states such that $\alpha_{m}=\alpha_{n}+\alpha_{f}$. This relation, combined with the momentum conservation condition implied by the delta function in the formulas for $\Gamma_{D, I}$ above, allows us to replace in Eq. (51) 


$$
Z_{m}=e^{-\beta p \cdot u+\alpha_{f}} Z_{n}
$$

which gives

$$
\Gamma_{I}=e^{-x} \Gamma_{D}
$$

or equivalently

$$
\left(1-n_{F}(x)\right) \Gamma_{I}=n_{F}(x) \Gamma_{D},
$$

where $n_{F}$ and $x$ are given in Eqs. (9) and (10), with $\mu=\alpha_{f} \beta$.

In order to establish the relation between $\Sigma_{i}$ and the total rate, we start from the defining Eq. (42). Inserting a complete set of states $\sum|n\rangle\langle n|$ between the fields $\bar{\eta}(y)$ and $\eta(z)$, we have

$$
\begin{aligned}
i \Sigma_{12}(z-y)_{\alpha \beta}= & \frac{1}{Z} \sum_{n, m} e^{-i\left(q_{m}-q_{n}\right) \cdot(z-y)} \times \\
& \left\langle m\left|\bar{\eta}_{\beta}(0)\right| n\right\rangle\left\langle n\left|\eta_{\alpha}(0)\right| m\right\rangle Z_{m},
\end{aligned}
$$

from which we immediately obtain

$$
i \bar{u}_{L}(p) \Sigma_{12}(p) u_{L}(p)=2 p^{0} \Gamma_{I}
$$

In similar fashion,

$$
\begin{aligned}
i \Sigma_{21}(z-y)_{\alpha \beta}= & \frac{-1}{Z} \sum_{n, m} e^{-i\left(q_{m}-q_{n}\right) \cdot(z-y)} \times \\
& \left\langle n\left|\eta_{\alpha}(0)\right| m\right\rangle\left\langle m\left|\bar{\eta}_{\beta}(0)\right| n\right\rangle Z_{n},
\end{aligned}
$$

so that

$$
i \bar{u}_{L}(p) \Sigma_{21}(p) u_{L}(p)=-2 p^{0} \Gamma_{D}
$$

Using these results in the relation $\Sigma_{i}=\frac{i}{2}\left(\Sigma_{21}-\Sigma_{12}\right)$ [Eq. (86] we finally obtain [19]

$$
\bar{u}_{L}(p) \Sigma_{i}(p) u_{L}(p)=-p^{0} \Gamma
$$

where 


$$
\Gamma=\Gamma_{I}+\Gamma_{D} .
$$

On the other hand, recall from Eq. (25) that the damping rate, determined as the imaginary part of the dispersion relation, is given by

$$
\gamma=-\frac{1}{\varepsilon_{r}} \bar{u}_{L} \Sigma_{i}\left(\varepsilon_{r}, P\right) u_{L} .
$$

where $\varepsilon_{r}$ is the (real part of the) true dispersion relation of the propagating mode, and $u_{L}$ is the corresponding spinor wave function. With the spinor $u_{L}$ thus chosen and with $p^{0}=\varepsilon_{r}$, $\Gamma$ in Eq. (60) has the interpretation of the total rate for all the processes involving the propagating fermion, so that Eqs. (59) and (25) establish the equality $\gamma=\Gamma$ with generality.

In conclussion, the damping rate, determined from the imaginary part of the dispersion relation, coincides with the total rate, provided the latter is calculated with the correct wave function of the propagating fermion mode. This result provides a clear and consistent interpretation of the absorptive part of the self-energy in terms of a single physical quantity. Further, since the damping rate is expressed in terms of transition probabilities, our work could serve to consider the problem of the gauge dependence of the fermion damping rate from a different point of view.

\section{Acknowledgement}

This work was partially supported by Grant DGAPA-IN100691 at the Universidad Nacional Autónoma de México, and by the US National Science Foundation Grant PHY-9320692 at the University of Puerto Rico. 


\section{REFERENCES}

[1] H. A. Weldon, Phys. Rev. D26 (1982)2789.

[2] With this convention for $\gamma$, the probability that the particle has not decayed behaves as $\sim e^{-\gamma t}$.

[3] A comprehensive review, with an extensive list of references, is given by N. P. Landsman and Ch. G. van Weert, Phys. Rep. 145 (1987)141. Some recent applications are reviewed by T. Altherr, preprint CERN-TH.6942/93, July 1993.

[4] For the particular application of this formalism to the case of a chiral fermion (e.g., a neutrino), see, for example, J. F. Nieves, in Proceedings of the IV Mexican School of Particles and Fields, edited by J. L. Lucio and A. Zepeda (World Scientific, Singapore, 1992), pp 283-319; J. C. D'Olivo, J. F. Nieves and M. Torres, Phys. Rev. D46 (1992)1172 and refences therein. See also J. F. Nieves, Phys. Rev. D42 (1990)4123.

[5] H. A. Weldon, Phys. Rev. D28 (1983)2007.

[6] L. P. Kadanoff and G. Baym, Quantum Statistical Mechanics, Frontiers in Physics, Lecture Note and Reprint Series (Benjamin Cummings, Reading, 1962), p. 39.

[7] The same problem has been examined exhaustively for scalar fields in Naoki Ashida, Hisao Nakkagawa, Akira Niegawa and Hiroshi Yokota, Ann. Phys. 215 (1992)315.

[8] U. Heinz, K. Kajantie and T. Toimela, Ann. Phys. 176 (1987)218.

[9] T. Altherr, E. Petitgirard and T. del Rio Gaztelurrutia, Phys. Rev. D47 (1993)703.

[10] E. Braaten and R. D. Pisarski, Phys. Rev. Lett. 64 (1990)1378; R. Baier, G. Kunstatter and D. Schiff, Phys. Rev. D45 (1992)4381; Anton Rebhan, ibid. 46 (1992)4779; R. Kobes, G. Kunstatter and K. Mak,ibid. 45 (1992)4632; H. Nakkagawa, A. Niégawa and B. Pire, Phys. Lett. B294 (1992)396. A recent review of the problem has been given by R. Baier, Bielefeld preprint BI-TP93/62, November 1993. 
[11] R.D. Pisarski, Phys. Rev. Lett. 63 (1989)1129; E. Braten and R. D. Pisarski, Nucl. Phys. B337 (1990)569; ibid. 339 (1990)310.

[12] M. H. Thoma and M. Gyulassy, Nucl. Phys. B351 (1991)491.

[13] See, for example, J. R. Schrieffer, Theory of Superconductivity, Revised Printing, Frontiers in Physics, Lecture Notes Series 20 (Benjamin Cummings, Reading, 1983), p. 118.

[14] H. A. Weldon, Phys. Rev. D40 (1989)2410.

[15] Since $f_{r}$ and $f_{i}$ are defined to be functions of the complex variable $\varepsilon$, they coincide with the real and imaginary parts of $f$ only for real values of that variable, but not otherwise.

[16] J. F. Nieves, Phys. Rev. D40 (1989)866.

[17] See, for example, D. A. Kirzhnits, Field Theoretical Methods in Many-Body Systems, (Pergamon Press, London, 1967), p. 260.

[18] See, for example, the book cited in Ref. [6], p. 36.

[19] Of course the same result follows also from the third formula in Eq. (8), using Eq. (56) and the relation between $\Gamma_{D}$ and $\Gamma_{I}$ given in Eq. (53). 


\section{Figure Captions}

Fig. 1. Self-energy diagram for the fermion $f$ due to the interaction $\lambda \bar{\psi}_{R} f_{L} \phi$. 